\title{
Current applications of robotics in spine surgery: a systematic review of the literature
}

\author{
Jacob R. Joseph, MD, Brandon W. Smith, MD, Xilin Liu, MD, and Paul Park, MD \\ Department of Neurosurgery, University of Michigan, Ann Arbor, Michigan
}

OBJECTIVE Surgical robotics has demonstrated utility across the spectrum of surgery. Robotics in spine surgery, however, remains in its infancy. Here, the authors systematically review the evidence behind robotic applications in spinal instrumentation.

METHODS This systematic review was conducted according to the Preferred Reporting Items for Systematic Reviews and Meta-Analysis (PRISMA) guidelines. Relevant studies (through October 2016) that reported the use of robotics in spinal instrumentation were identified from a search of the PubMed database. Data regarding the accuracy of screw placement, surgeon learning curve, radiation exposure, and reasons for robotic failure were extracted.

RESULTS Twenty-five studies describing 2 unique robots met inclusion criteria. Of these, 22 studies evaluated accuracy of spinal instrumentation. Although grading of pedicle screw accuracy was variable, the most commonly used method was the Gertzbein and Robbins system of classification. In the studies using the Gertzbein and Robbins system, accuracy (Grades A and B) ranged from $85 \%$ to 100\%. Ten studies evaluated radiation exposure during the procedure. In studies that detailed fluoroscopy usage, overall fluoroscopy times ranged from 1.3 to 34 seconds per screw. Nine studies examined the learning curve for the surgeon, and 12 studies described causes of robotic failure, which included registration failure, soft-tissue hindrance, and lateral skiving of the drill guide.

CONCLUSIONS Robotics in spine surgery is an emerging technology that holds promise for future applications. Surgical accuracy in instrumentation implanted using robotics appears to be high. However, the impact of robotics on radiation exposure is not clear and seems to be dependent on technique and robot type.

https://thejns.org/doi/abs/10.3171/2017.2.FOCUS16544

KEY WORDS surgical robotics; spine surgery; Mazor; Renaissance; SpineAssist; ROSA

$\mathrm{W}$ HEREAS robotics has grown to be commonplace in other arenas, robotics in spine surgery remains a relative novelty. However, there is a burgeoning body of evidence to suggest that robotics may develop into a part of everyday spine surgery practice. Historically, freehand pedicle screw placement without image guidance has resulted in high rates of inaccuracy. With the addition of fluoroscopy, accuracy rates have improved. However, the risk of nerve and (less commonly) vascular injury continues to exist. In addition to these patient-related risks, there has been increasing concern about the risk of radiation exposure for the surgeon and staff with the use of intraoperative fluoroscopy. ${ }^{34}$ Robotics could potentially offer solutions to both of these concerns while improving surgical ergonomics and enhancing surgical dexterity, and thereby could potentially improve patient outcomes. . $^{13,26,39,41}$ We systematically reviewed the current body of evidence for robotics as it applies to spinal instrumentation. Accuracy of robotics, the surgeon's learning curve, radiation exposure, and reasons for robotic failure were examined in detail.

\section{Methods}

\section{Literature Search and Inclusion Criteria}

This systematic review was conducted according to Preferred Reporting Items for Systematic Reviews and Meta-Analyses (PRISMA) guidelines. ${ }^{23}$ We identified relevant articles published up to October 26, 2016, through a search of the PubMed database. The initial search strat-

ABBREVIATIONS FT = fluoroscopy time; GO-LIF = guided oblique lumbar interbody fusion; GRS = Gertzbein and Robbins system; PLIF = posterior lumbar interbody fusion; RCT = randomized controlled trial. 
egy for publications was performed using the search terms (robot[tiab] or robotic[tiab]) AND (pedicle or spine or spinal or vertebral or vertebrae) AND (surgery or surgical[text word]). In addition, manual checks through the reference lists were performed. Only articles published in the English language were included. After the initial search, titles and abstracts were reviewed. Finally, each article that met criteria underwent full-text review. Articles that did not have full text available were excluded. Articles that did not evaluate robotic placement of spinal instrumentation were excluded. Articles detailing the use of robotics only for surgical exposure were excluded. Case reports, technique papers, cadaveric studies, and animal studies were excluded.

\section{Data Extraction and Outcome Measures}

We extracted the following data from each report: primary author name, senior author name, date of publication, study type, method of data collection (prospective or retrospective), comparison group, robot type, spine segments involved, approach, instrumentation performed, number of patients, and number of screws placed. Data regarding the accuracy of screw placement, learning curve, radiation exposure, and reasons for robotic failure were extracted. Risk of bias was evaluated for each study at the time of data extraction, and it was noted that there was a risk for publication bias in the present study. Accuracy of screw placement was the primary outcome of the study, and secondary outcomes were radiation exposure and determination of a surgical learning curve.

\section{Results \\ Study Selection}

A total of 402 articles were identified through the database searches and underwent review of titles and abstracts. Three hundred six studies that did not involve robotics for spine surgery were excluded. The remaining 96 articles then underwent abstract review. Thirty-seven articles were subsequently excluded; 59 articles were deemed eligible for full-text review. After full-text review, 35 articles were excluded. Reasons for exclusion included study type, duplicate series, studies not involving spinal instrumentation, foreign language, and robotics only used for exposure. One additional study was identified through manual review of reference lists. A total of 25 studies therefore met the criteria (Table 1); of these, 18 studies were retrospective and 7 were prospective. Four randomized controlled trials (RCTs) were identified. The search flow diagram is shown in Fig. 1.

\section{Type of Robot}

Our search revealed 2 robots that have been used for spinal instrumentation. Twenty-four studies evaluated robots produced by Mazor Surgical Technologies; ;,2, $5,6,9-15,17,22,24,25,27-32,35-37$ these included the SpineAssist as well as the Renaissance robot. One study evaluated the ROSA robot (Medtech). ${ }^{21}$ Robots that were exclusively used for exposure, such as for anterior lumbar interbody fusion procedures, were excluded as defined in the Methods. ${ }^{18}$

\section{Accuracy of Spinal Instrumentation}

A total of 22 studies evaluated accuracy of spinal instru-

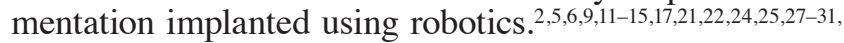
35-37 Twenty-one of these studies used variations of the robot developed by Mazor, whereas 1 study evaluated the ROSA robot. Twenty-one studies evaluated pedicle screw accuracy, whereas Dreval et al. evaluated both pedicle screws and guided oblique lumbar interbody fusion (GOLIF) screws, ${ }^{6}$ and Bederman et al. evaluated the accuracy of S2-alar-iliac screw placement. ${ }^{2}$

Sukovich et al. first reported on the Mazor system in $2006 .{ }^{35}$ They retrospectively evaluated 14 patients in whom 98 pedicle screws were placed. A combination of open and minimally invasive techniques were used. They found that $96 \%$ of the screws were within $1-2 \mathrm{~mm}$ of the planned trajectory, and they found no instances of breaching of the pedicle, although these authors did not detail their method of grading pedicle screw accuracy. Pechlivanis et al. ${ }^{25}$ also retrospectively reviewed accuracy of the Mazor robot during minimally invasive posterior lumbar interbody fusion (PLIF), by using the postoperative CT-based Gertzbein and Robbins system (GRS) to classify pedicle screw accuracy. ${ }^{7,25}$ In the GRS, screws completely within the pedicle are Grade A; a breach of $<2 \mathrm{~mm}$ is Grade B; a breach of 2 to $<4 \mathrm{~mm}$ is Grade $\mathrm{C}$; a breach of 4 to $<6 \mathrm{~mm}$ is Grade $\mathrm{D}$; and a breach of $>6 \mathrm{~mm}$ is Grade E. In this system, both Grades A and B are deemed acceptable. Pechlivanis et al. found that of a total of 122 screws that were able to be graded in 31 patients, $108(88.5 \%)$ were GRS Grade A, $13(10.7 \%)$ were GRS Grade B, and $1(0.8 \%)$ was GRS Grade D.

Devito et al. have performed the largest retrospective study to date, evaluating 635 patients in whom 3271 pedicle screws were placed. ${ }^{5}$ Of these screws, $98 \%$ were deemed acceptable by fluoroscopy. A subgroup of 646 screws were evaluated with postoperative CT. In this subanalysis, 577 (89.3\%) were GRS Grade A; 58 (9.0\%) were GRS Grade B; 9 (1.4\%) were GRS Grade C; and 2 $(0.3 \%)$ were GRS Grade D. Hu and colleagues published a series of papers retrospectively assessing pedicle screw accuracy ${ }^{9-11}$ In their first paper, ${ }^{10}$ accuracy was evaluated using fluoroscopy in 95 patients with 960 screws placed using the Mazor robot (note that accuracy was not graded in all 102 patients; in the other 7 the screw placement was aborted). Of these, 949 (98.9\%) were deemed to be accurate. In a follow-up study looking specifically at 9 patients with spinal column tumors, these authors did not mention any misplacements, although accuracy was not graded specifically.

Dreval et al. evaluated 14 patients who underwent minimally invasive pedicle screw placement. ${ }^{6}$ These authors did not define accuracy, although they stated that all patients had good results. Onen et al. evaluated 27 patients undergoing placement of 136 pedicle screws. ${ }^{24}$ In their cohort, 124 (91.2\%) were GRS Grade A, 10 (7.4\%) were GRS Grade B, and 2 (1.5\%) were GRS Grade C. Schatlo et al. published a series of 258 cases with 1265 pedicle screws, and found that $96.2 \%$ were acceptably placed (GRS Grade A or B). ${ }^{29}$ Similarly, van Dijk et al. evaluated 112 patients with 494 robotically placed screws. ${ }^{37}$ A $97.9 \%$ rate of acceptably placed screws (GRS Grade A or B) was noted. 
TABLE 1. Summary of 25 articles included in systematic literature review related to robotics in spine surgery

\begin{tabular}{|c|c|c|c|c|c|c|c|c|}
\hline \multirow[b]{2}{*}{ Authors \& Year } & \multirow[b]{2}{*}{$\begin{array}{c}\text { Data } \\
\text { Collection }\end{array}$} & \multirow[b]{2}{*}{$\begin{array}{l}\text { Study } \\
\text { Type }\end{array}$} & \multirow[b]{2}{*}{$\begin{array}{l}\text { Comparison } \\
\text { Group }\end{array}$} & \multirow[b]{2}{*}{$\begin{array}{l}\text { Robot } \\
\text { Type }\end{array}$} & \multirow[b]{2}{*}{ Instrumentation } & \multicolumn{3}{|c|}{ Screws } \\
\hline & & & & & & $\begin{array}{l}\text { No. of } \\
\text { Pts }\end{array}$ & $\begin{array}{c}\text { No. Screws } \\
\text { Placed }\end{array}$ & $\begin{array}{c}\text { Accuracy } \\
(\%)\end{array}$ \\
\hline Barzilay et al., 2006 & Prospective & Case series & None & Mazor & Pedicle screw & 15 & & \\
\hline Bederman et al., 2016 & Retrospective & Case series & None & Mazor & S2-alar-iliac screw & 14 & 31 & 100 \\
\hline Devito et al., 2010 & Retrospective & Case series & None & Mazor & Pedicle screw & 635 & 3271 & 98.3 \\
\hline Dreval et al., 2014 & Retrospective & Case series & None & Mazor & GO-LIF \& pedicle screw & 50 & & \\
\hline Hu et al., 2013 & Retrospective & Case series & None & Mazor & Pedicle screw & 102 & 1085 & 98.9 \\
\hline Hu \& Lieberman, 2014 & Retrospective & Case series & None & Mazor & Pedicle screw & 162 & & \\
\hline Hu et al., 2015 & Retrospective & Case series & None & Mazor & Pedicle screw & 9 & & \\
\hline Hyun et al., 2016 & Prospective & $\mathrm{RCT}$ & Freehand & Mazor & Pedicle screw & 30 & 130 & 100 \\
\hline Kantelhardt et al., 2011 & Retrospective & Case series & Freehand & Mazor & Pedicle screw & 55 & 250 & 94.5 \\
\hline Keric et al., 2017 & Retrospective & Comparative & Freehand & Mazor & Pedicle screw & 66 & 341 & 90.0 \\
\hline Kim et al., 2016 & Prospective & $\mathrm{RCT}$ & Freehand & Mazor & Pedicle screw & 37 & 158 & 99.4 \\
\hline Kuo et al., 2016 & Retrospective & Case series & None & Mazor & Pedicle screw & 64 & 317 & 98.7 \\
\hline Lonjon et al., 2016 & Prospective & Matched cohort & Freehand & ROSA & Pedicle screw & 10 & 40 & 97.2 \\
\hline Macke et al., 2016 & Retrospective & Case series & None & Mazor & Pedicle screw & 50 & 662 & 92.7 \\
\hline Onen et al., 2014 & Retrospective & Case series & None & Mazor & Pedicle screw & 27 & 136 & 98.6 \\
\hline Pechlivanis et al., 2009 & Retrospective & Case series & None & Mazor & Pedicle screw & 31 & 133 & 99.2 \\
\hline Ringel et al., 2012 & Prospective & RCT & Freehand & Mazor & Pedicle screw & 30 & 146 & 85.0 \\
\hline Roser et al., 2013 & Prospective & RCT & $\begin{array}{l}\text { Freehand, stan- } \\
\text { dard navigation }\end{array}$ & Mazor & Pedicle screw & 18 & 72 & 99.0 \\
\hline Schatlo et al., 2014 & Retrospective & Matched cohort & Freehand & Mazor & Pedicle screw & 55 & 244 & 91.4 \\
\hline Schatlo et al., 2015 & Retrospective & Case series & None & Mazor & Pedicle screw & 258 & 1265 & 96.2 \\
\hline Schizas et al., 2012 & Prospective & Matched cohort & Freehand & Mazor & Pedicle screw & 11 & 64 & 95.3 \\
\hline Sensakovic et al., 2016 & Retrospective & Matched cohort & None & Mazor & Pedicle screw & 34 & & \\
\hline Sukovich et al., 2006 & Retrospective & Case series & None & Mazor & Pedicle screw & 14 & 98 & 96.0 \\
\hline Tsai et al., 2016 & Retrospective & Case series & None & Mazor & Pedicle screw & 35 & 176 & 98.9 \\
\hline van Dijk et al., 2015 & Retrospective & Case series & None & Mazor & Pedicle screw & 112 & 494 & 97.9 \\
\hline
\end{tabular}

Pts = patients.

The blank cells in the last 2 columns denote that those values were not reported in the studies cited.

Recently, Macke et al. evaluated the Mazor robot in the treatment of adolescent idiopathic scoliosis specifically. ${ }^{22}$ They reported a series of 48 patients with 662 screws in total ( 2 patients were eliminated from accuracy grading due to inadequate CT scans). They found a $92.7 \%$ acceptable placement rate (GRS Grade A or B). Of the 48 misplaced screws, 30 were GRS Grade C, 10 were GRS Grade D, and 8 were GRS Grade E.

Kuo et al. evaluated the use of secondary registration on pedicle screw accuracy when using the Mazor robot. ${ }^{17}$ In this protocol, the authors placed a K-wire using the robot, and then reregistered to the guidance system. Any deviation of $>3 \mathrm{~mm}$ was repositioned. The authors then graded accuracy when using biplanar fluoroscopy. Three hundred seventeen K-wires were placed using this system. Of these, $19(6.0 \%)$ were noted to have a $>3-\mathrm{mm}$ deviation and underwent repositioning. After repositioning, $15 \mathrm{im}-$ proved to having a $<3-\mathrm{mm}$ deviation, and the remaining 4 required manual adjustment (98.7\% final accuracy rate). The authors subsequently validated this accuracy data by using CT-based accuracy grading systems such as the
GRS. They concluded that secondary registration increases the accuracy rate of robotic pedicle screw placement.

Seven comparative studies evaluating the Mazor robot as opposed to conventional freehand technique with fluoroscopy assistance were identified; this included 3 RCTs. Ringel et al. provided the first RCT in 2012. ${ }^{27}$ They randomly assigned 60 patients evenly into either percutaneous robot pedicle screw placement or the conventional open freehand technique. With the robotic arm, 146 screws were attempted, with an $85 \%$ rate of acceptably placed screws (GRS Grade A or B). With the freehand method, a 93\% rate of acceptably placed screws was noted. The authors concluded that robotic placement of pedicle screws was inferior to conventional techniques. Hyun et al. performed a similarly designed study, again with 30 patients in each group. ${ }^{12}$ They found that all robotically placed screws were acceptably placed. In the freehand group, there was a $98.6 \%$ accuracy rate. In addition, the freehand group had 1 violation of the proximal facet, whereas there were none seen in the robotic group. Kim et al. conducted an RCT of minimally invasive PLIF performed using the 


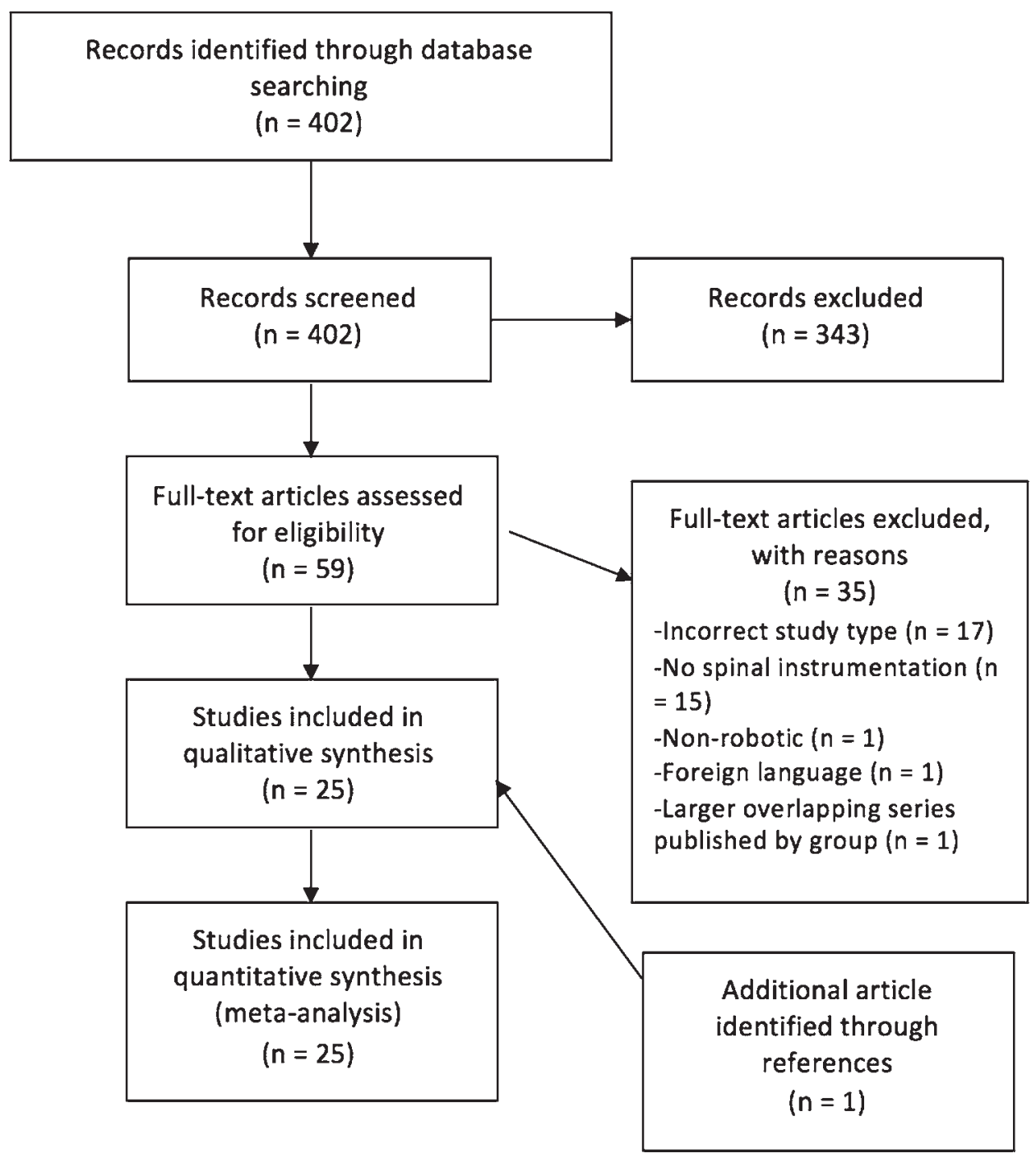

FIG. 1. Flow diagram showing selection of the studies for this systematic review of the use of robotics in spinal instrumentation.

Mazor robot compared with open freehand techniques for PLIF. ${ }^{15}$ They found a $99.4 \%$ accuracy rate when using the robot, and a $99.4 \%$ accuracy rate with conventional freehand techniques. However, there were no proximal joint violations with the robot, and $13(15.9 \%)$ violations with the freehand technique. Kantelhardt et al. compared robotic screw placement (both open and percutaneous) with freehand techniques in a nonrandomized, retrospective fashion..$^{13}$ They saw significantly better accuracy when using the robot (94.5\% vs $91.5 \%$ freehand), although there was no difference in accuracy in open robotic or percutaneous robotic fusion.

A prospective case-matched study by Schizas et al. found that robotically placed screws had a $95.3 \%$ accuracy rate compared with a $92.2 \%$ accuracy rate with conventionally placed screws, and concluded that there was no difference between the 2 techniques. ${ }^{31}$ Schatlo et al. also performed a retrospective case-matched study comparing robotically placed pedicle screws (both open and percutaneous) with open freehand screws. ${ }^{30}$ They found a $91.4 \%$ accuracy rate with Mazor, and an $87.1 \%$ accuracy rate with open techniques. Six robotically placed screws required manual revision during the initial operation, and 1 freehand screw required postoperative revision due to misplacement causing radiculopathy. Keric et al. performed a retrospective cohort study in patients with spondylodiscitis between percutaneous robotically placed pedicle screws and open freehand procedures. ${ }^{14}$ They found that robotically placed screws were significantly more accurate, and were less likely to require revision for misplacement or loosening.

Roser et al. performed an RCT using 3 arms: freehand instrumentation, standard neuronavigation, and robotic screw placement using the Mazor robot. ${ }^{28}$ They found a 99\% accuracy rate (GRS A) with robotically placed screws. Standard navigation resulted in $92 \%$ accuracy (GRS A), and freehand techniques resulted in $97.5 \%$ accuracy. Of note, the authors defined accuracy as only GRS A, which is dissimilar to other studies that also included GRS $\mathrm{B}$ as accurate. When including GRS B screws, accuracy 
rates were $99 \%$ with robotics, $97.2 \%$ with navigation, and $100 \%$ with freehand. Statistical evaluation was not performed in this study due to the small sample size of only 18 patients treated with robotics, 9 with navigation, and 10 with the freehand method.

The Mazor robot has also been evaluated for procedures other than placement of pedicle screws. Bederman et al. evaluated accuracy of the placement of 31 S2-alariliac screws in 14 patients. ${ }^{2}$ They found that all screws were accurate, with no breach of the anterior sacrum. Dreval et al. evaluated transpedicular, transdiscal screws in association with GO-LIF. ${ }^{6}$ Thirty-six patients and 72 screws were evaluated for this procedure. Accuracy was not directly graded, but 1 patient did require revision due to poor purchase.

Lonjon et al. performed the only study evaluating the ROSA robot. ${ }^{21}$ They performed a prospective casematched study comparing robotically placed screws to open freehand techniques. Thirty-six screws were robotically placed, with a $97.2 \%$ accuracy rate, and 50 screws were placed freehand with a $92 \%$ accuracy rate. An additional 4 screws were attempted robotically, but were unable to be placed due to technical difficulties.

\section{Radiation Exposure}

Ten studies evaluated radiation exposure in conjunction with robotic spinal instrumention. ${ }^{12-15,21,24,27,28,31,32}$ This included 5 studies comparing robotic procedures with conventional open procedures. Kantelhardt et al. found that robotically placed screws had a mean fluoroscopy time (FT) of 34 seconds per screw, whereas open freehand screws had a mean FT of 77 seconds. ${ }^{13}$ There was no difference between percutaneous robotically placed screws and open robotically placed screws. Hyun et al. also found that the mean FT per screw was significantly lower with the robot (3.5 seconds vs 13.3 seconds). ${ }^{12}$ Similarly, the robotically placed screws had decreased radiation output in millisieverts $(\mathrm{mSv})$ when compared with freehand placement $(0.13 \mathrm{mSv}$ vs 0.27 $\mathrm{mSv}$ ). Keric et al. also found that FT was significantly lower with robotically placed screws. ${ }^{14}$ However, Ringel et al. found that total intraoperative FT was similar between freehand and robotically placed screws. ${ }^{27}$ Schizas et al. also found that radiation times were similar (16.7 seconds per screw robotically vs 14.2 seconds per screw freehand). ${ }^{31}$ Onen et al. described an FT of 1.3 seconds per screw, whereas Kim et al. described a total FT of 20.4 seconds. ${ }^{15,24}$ Roser et al. evaluated both standard navigation and freehand techniques in comparison with robotically placed screws. ${ }^{28}$ They reported that FT and radiation dosage were lowest in the standard navigation group, followed by the robotic group. Radiation exposure was highest in the freehand group.

Sensakovic et al. evaluated a new low-dose radiation $\mathrm{CT}$ protocol for patients undergoing pediatric idiopathic scoliosis deformity correction performed using the Mazor robot. ${ }^{32}$ In this protocol, patients either underwent traditional preoperative CT or low-dose CT. Dose reductions for the preoperative CT were $6.55 \mathrm{mSv}$ in patients with a body mass index $<25$, and $9.3 \mathrm{mSv}$ for patients with a body mass index of 25-35. The authors reported that im- ages were adequate for robotic screw placement, although accuracy of screw placement was not graded postoperatively.

Lonjon et al. evaluated radiation exposure during placement of pedicle screws performed using the ROSA robot as compared with freehand placement. ${ }^{21}$ They found that total FT was significantly longer when using the robot (1.23 minutes vs 0.4 minute). The FT per screw was 25 seconds when using ROSA, and 10 seconds when using freehand techniques. In that study, intraoperative fluoroscopy was used for registration and planning purposes.

\section{Learning Curve}

Eight studies evaluated the learning curve for spinal instrumentation placement performed using robotics. ${ }^{5,10,12,15 \text {, }}$ $22,24,27,29$ Devito et al. found that the ability to place screws robotically was $83.7 \%$ in their total cohort, but increased to a $90.8 \%$ execution rate when they looked at their most recent procedures. ${ }^{5}$ In addition, time per screw placement decreased from 13.5 minutes for single-level cases (4 minutes per screw in multilevel cases) to 10.6 minutes ( 2 minutes in multilevel cases). Hu et al. evaluated the learning curve specifically, and found that after 30 procedures, the rate of successful placement increased and there was a decreased need for conversion to manual techniques. ${ }^{10}$ There were no differences in rates of malpositioning, and the learning effect plateaued after the initial 30 patients. Onen et al. saw a decrease in both FT (from 1.8 seconds per screw to 0.9 seconds per screw) and time for screw placement (from 15.5 minutes to 8.6 minutes) over the course of their experience when comparing their initial 13 patients to their subsequent 14 patients. ${ }^{24}$ Hyun et al. similarly found a decrease in time for screw placement (from 5.5 minutes to 4.0 minutes) and FT (from 4.1 seconds to 2.9 seconds). ${ }^{12}$ Kim et al. found a decrease in total FT from 27.5 seconds to 18.5 seconds. ${ }^{15}$ Schatlo et al. graded the accuracy of screw placement in 13 surgeons. ${ }^{29}$ For each surgeon, accuracy was graded in 5-case increments. The investigators found that misplacement rates peaked between 5 and 25 surgeries, and then steadily declined. Macke et al. also found a decrease in misplacements when comparing the first and last third of their cohort $(9.6 \%$ vs 7.4\%). ${ }^{22}$ However, Ringel et al. stated that accuracy did not improve over the course of their study, although no specific data were provided. ${ }^{27}$

\section{Mechanisms of Robotic Failure}

Twelve studies evaluated reasons for failure of the robot during spine instrumentation. . $^{1,9,17,21,22,24,25,27,28,30,35}$ Reasons for aborting the robotic procedure included failure of registration software and failure to obtain adequate fluoroscopic images. In several studies, soft-tissue pressure on the guiding arm led to inaccurate placement. ${ }^{1,27}$ Other reasons for failure included the inability to adequately obtain surgically the necessary angles determined by the registration software. Other studies describe difficulties with keeping the drill guide in position on the slope of the facet, causing a lateral and inferior deviation. ${ }^{17,27,30}$ Macke et al. found that patients who had preoperative CTs performed in a prone position had a screw misplacement rate of $2.4 \%$, 
compared with a misplacement rate of $7.6 \%$ in patients whose preoperative CTs were performed in the standard supine position..$^{22}$

\section{Discussion}

Appropriate instrumentation to supplement bony fusion remains critically important in spine surgery. Recently, surgical robots have been developed and studied for their ability to improve spinal instrumentation techniques. The Mazor robot (SpineAssist or Renaissance) has been the most extensively studied. It is a miniature bone-mounted robot that has 6 degrees of freedom. ${ }^{1,20,35}$ A preoperative $\mathrm{CT}$ is used to plan trajectories, and intraoperative fluoroscopy is used to register the images. The robot then guides the surgeon to the appropriate trajectory. The ROSA robot includes a mobile floor-fixed base attached to a robotic arm with 6 degrees of freedom. A second mobile base has a navigation camera mounted to it. The ROSA is an imageguided device and uses an iliac pin for a reference point. Either intraoperative fluoroscopy or intraoperative CT can be used for planning., ${ }^{4,1921}$ Currently, both robots only have applications in placement of screws for spinal instrumentation; no further applications have been described. Comparisons between ROSA and Mazor are summarized in Table 2.

Robotics in spine surgery offers the advantage of precision and the removal of human manual error. In this review, all studies that evaluated accuracy of screw placement (both pedicle screws and S2-alar-iliac screws) showed that the accuracy rates were high. Most comparative studies demonstrated that robotics provides an advantage to traditional freehand placement. However, 1 RCT did show that there was a decrease in accuracy with screw placement using the Mazor robot. ${ }^{27}$ Overall, the accuracy data suggest that screw placement with the Mazor robot is safe. Although only 1 study evaluated the ROSA robot, it also appeared to show that pedicle screw placement with this device is safe. ${ }^{21}$ In addition to accuracy through the pedicle, some studies suggested that using robotics for pedicle screw placement allows the surgeon to avoid violating the proximal facet joint, which may provide biomechanical advantages that could preclude the development of adjacent-segment disease. ${ }^{16}$

There are no large-scale studies to date that have directly compared robotic guidance of pedicle screws with image guidance. Roser et al. did publish a preliminary series evaluating image guidance in comparison with both robotics and freehand placement. ${ }^{28}$ Because of inadequate power, with only 37 total patients among the 3 treated groups, they were unable to analyze their results. Image guidance provided using intraoperative cone-beam CT has already been shown to be superior to freehand placement. ${ }^{33,38}$ As mentioned previously, the ROSA robot does incorporate image guidance into its system, in addition to having the robotic arm.

Radiation exposure to the surgeon and operating room staff is a concern, particularly in minimally invasive procedures. ${ }^{3,8,40}$ One potential advantage of robotic spinal instrumentation is to minimize reliance on intraoperative fluoroscopy. Findings for FT in the Mazor robot
TABLE 2. Comparison of Mazor and ROSA robotics systems

\begin{tabular}{cll}
\hline \multirow{2}{*}{ Feature } & \multicolumn{2}{c}{ Robotics System } \\
\cline { 2 - 3 } & Mazor $^{*}$ & ROSA \\
\hline Degrees of freedom & 6 & 6 \\
\hline Mount & Bone & Floor \\
\hline Preop CT required & Yes & No \\
\hline Instrument tracking & No & Yes \\
\hline FDA approval & Yes & Yes \\
\hline * Renaissance robot. & &
\end{tabular}

were variable, ranging from 1.3 seconds to 34 seconds per screw, probably due to surgeon variability. ${ }^{13,24}$ In comparative studies, results suggested that the Mazor robot allowed for FT similar to or lower than FT for open freehand procedures. However, evaluation of the ROSA robot showed a significant increase in FT when compared with freehand. ${ }^{21}$ It should be noted that this study used fluoroscopy for registration and planning. Studies of the ROSA robot performed using intraoperative CT were not available.

Although surgical robotics is promising, it is still clearly in its nascent stage. Execution rates for robotic procedures are still not as high as would be ideal. Issues such as soft-tissue pressure on the robotic arm were cited several times in the literature as causing deviations. ${ }^{1,27}$ In addition, slipping of the guide down the slope of the facet was commonly referred to as a major concern. ${ }^{17,27,30}$

There were several limitations to the present study. An inherent risk of publication bias exists with the literature, as previously mentioned. In addition, there were inconsistencies among the studies in their reporting of pedicle screw accuracy. Although several studies used CT-based methods, some evaluated accuracy using intraoperative fluoroscopy. Similarly, methods of evaluating radiation exposure varied among studies. Finally, because most studies used the Mazor robot, the results presented here may not truly represent surgical robotics as a whole, but rather the experience with a particular robot.

\section{Conclusions}

Robotics in spine surgery is a new technology that holds promise for future applications. Currently, placement of pedicle screws with robotics appears to be safe, and accuracy appears to be superior to freehand placement, although the data are not conclusive. Radiation exposure is dependent on the type of robot, and from the current literature we were unable to definitively confirm if there is a significant benefit to robotics for this concern. Studies comparing robotics with image guidance are currently lacking. More research is necessary to identify the ideal role for robotics in spinal instrumentation.

\section{References}

1. Barzilay Y, Liebergall M, Fridlander A, Knoller N: Miniature robotic guidance for spine surgery-introduction of a novel system and analysis of challenges encountered during the 
clinical development phase at two spine centres. Int J Med Robot 2:146-153, 2006

2. Bederman SS, Hahn P, Colin V, Kiester DP, Bhatia NN: Robotic guidance for S2-alar-iliac screws in spinal deformity correction. Clin Spine Surg [epub ahead of print], 2016

3. Bindal RK, Glaze S, Ognoskie M, Tunner V, Malone $\mathrm{R}$, Ghosh S: Surgeon and patient radiation exposure in minimally invasive transforaminal lumbar interbody fusion. J Neurosurg Spine 9:570-573, 2008

4. Chenin L, Peltier J, Lefranc M: Minimally invasive transforaminal lumbar interbody fusion with the ROSA(TM) Spine robot and intraoperative flat-panel CT guidance. Acta Neurochir (Wien) 158:1125-1128, 2016

5. Devito DP, Kaplan L, Dietl R, Pfeiffer M, Horne D, Silberstein B, et al: Clinical acceptance and accuracy assessment of spinal implants guided with SpineAssist surgical robot: retrospective study. Spine (Phila Pa 1976) 35:2109-2115, 2010 [Erratum in Spine (Phila Pa 1976) 36:91]

6. Dreval ON, Rynkov IP, Kasparova KA, Bruskin A, Aleksandrovskii V, Zil'bernshtein V: [Results of using Spine Assist Mazor in surgical treatment of spine disorders.] Zh Vopr Neirokhir Im N N Burdenko 78:14-20, 2014 (Russian)

7. Gertzbein SD, Robbins SE: Accuracy of pedicular screw placement in vivo. Spine (Phila Pa 1976) 15:11-14, 1990

8. Grelat M, Zairi F, Quidet M, Marinho P, Allaoui M, Assaker R: [Assessment of the surgeon radiation exposure during a minimally invasive TLIF: Comparison between fluoroscopy and O-arm system.] Neurochirurgie 61:255$259,2015(\mathrm{Fr})$

9. Hu X, Lieberman IH: What is the learning curve for roboticassisted pedicle screw placement in spine surgery? Clin Orthop Relat Res 472:1839-1844, 2014

10. Hu X, Ohnmeiss DD, Lieberman IH: Robotic-assisted pedicle screw placement: lessons learned from the first 102 patients. Eur Spine J 22:661-666, 2013

11. Hu X, Scharschmidt TJ, Ohnmeiss DD, Lieberman IH: Robotic assisted surgeries for the treatment of spine tumors. Int J Spine Surg 9:1, 2015

12. Hyun SJ, Kim KJ, Jahng TA, Kim HJ: Minimally invasive, robotic vs. open fluoroscopic-guided spinal instrumented fusions - a randomized, controlled trial. Spine (Phila Pa 1976) [epub ahead of print], 2016

13. Kantelhardt SR, Martinez R, Baerwinkel S, Burger R, Giese A, Rohde V: Perioperative course and accuracy of screw positioning in conventional, open robotic-guided and percutaneous robotic-guided, pedicle screw placement. Eur Spine J 20:860-868, 2011

14. Keric N, Eum DJ, Afghanyar F, Rachwal-Czyzewicz I, Renovanz M, Conrad J, et al: Evaluation of surgical strategy of conventional vs. percutaneous robot-assisted spinal transpedicular instrumentation in spondylodiscitis. J Robot Surg 11:17-25, 2017

15. Kim HJ, Jung WI, Chang BS, Lee CK, Kang KT, Yeom JS: A prospective, randomized, controlled trial of robot-assisted vs freehand pedicle screw fixation in spine surgery. Int J Med Robot [epub ahead of print], 2016

16. Kim HJ, Kang KT, Park SC, Chang BS, Lee CK, Yeom JS, et al: Biomechanical advantages of robot-assisted pedicle screw fixation in posterior lumbar interbody fusion compared to free-hand technique in a prospective randomized controlled trial-perspective for patient-specific finite element analysis. Spine J [epub ahead of print], 2016

17. Kuo KL, Su YF, Wu CH, Tsai CY, Chang CH, Lin CL, et al: Assessing the intraoperative accuracy of pedicle screw placement by using a bone-mounted miniature robot system through secondary registration. PLoS One 11:e0153235, 2016
18. Lee Z, Lee JY, Welch WC, Eun D: Technique and surgical outcomes of robot-assisted anterior lumbar interbody fusion. J Robot Surg 7:177-185, 2013

19. Lefranc M, Peltier J: Evaluation of the ROSA ${ }^{\mathrm{TM}}$ Spine robot for minimally invasive surgical procedures. Expert Rev Med Devices 13:899-906, 2016

20. Lieberman IH, Togawa D, Kayanja MM, Reinhardt MK, Friedlander A, Knoller N, et al: Bone-mounted miniature robotic guidance for pedicle screw and translaminar facet screw placement: Part I-Technical development and a test case result. Neurosurgery 59:641-650, 2006

21. Lonjon N, Chan-Seng E, Costalat V, Bonnafoux B, Vassal M, Boetto J: Robot-assisted spine surgery: feasibility study through a prospective case-matched analysis. Eur Spine J 25:947-955, 2016

22. Macke JJ, Woo R, Varich L: Accuracy of robot-assisted pedicle screw placement for adolescent idiopathic scoliosis in the pediatric population. J Robot Surg 10:145-150, 2016

23. Moher D, Shamseer L, Clarke M, Ghersi D, Liberati A, Petticrew M, et al: Preferred reporting items for systematic review and meta-analysis protocols (PRISMA-P) 2015 statement. Syst Rev 4:1, 2015

24. Onen MR, Simsek M, Naderi S: Robotic spine surgery: a preliminary report. Turk Neurosurg 24:512-518, 2014

25. Pechlivanis I, Kiriyanthan G, Engelhardt M, Scholz M, Lücke S, Harders A, et al: Percutaneous placement of pedicle screws in the lumbar spine using a bone mounted miniature robotic system: first experiences and accuracy of screw placement. Spine (Phila Pa 1976) 34:392-398, 2009

26. Renda A, Vallancien G: Principles and advantages of robotics in urologic surgery. Curr Urol Rep 4:114-118, 2003

27. Ringel F, Stüer C, Reinke A, Preuss A, Behr M, Auer F, et al: Accuracy of robot-assisted placement of lumbar and sacral pedicle screws: a prospective randomized comparison to conventional freehand screw implantation. Spine (Phila Pa 1976) 37:E496-E501, 2012

28. Roser F, Tatagiba M, Maier G: Spinal robotics: current applications and future perspectives. Neurosurgery 72 (Suppl 1):12-18, 2013

29. Schatlo B, Martinez R, Alaid A, von Eckardstein K, Akhavan-Sigari R, Hahn A, et al: Unskilled unawareness and the learning curve in robotic spine surgery. Acta Neurochir (Wien) 157:1819-1823, 2015

30. Schatlo B, Molliqaj G, Cuvinciuc V, Kotowski M, Schaller $\mathrm{K}$, Tessitore E: Safety and accuracy of robot-assisted versus fluoroscopy-guided pedicle screw insertion for degenerative diseases of the lumbar spine: a matched cohort comparison. $\mathbf{J}$ Neurosurg Spine 20:636-643, 2014

31. Schizas C, Thein E, Kwiatkowski B, Kulik G: Pedicle screw insertion: robotic assistance versus conventional C-arm fluoroscopy. Acta Orthop Belg 78:240-245, 2012

32. Sensakovic WF, O'Dell MC, Agha A, Woo R, Varich L: CT radiation dose reduction in robot-assisted pediatric spinal surgery. Spine (Phila Pa 1976) [epub ahead of print], 2016

33. Shin MH, Hur JW, Ryu KS, Park CK: Prospective comparison study between the fluoroscopy-guided and navigation coupled with $\mathrm{O}$-arm-guided pedicle screw placement in the thoracic and lumbosacral spines. J Spinal Disord Tech 28:E347-E351, 2015

34. Srinivasan D, Than KD, Wang AC, La Marca F, Wang PI, Schermerhorn TC, et al: Radiation safety and spine surgery: systematic review of exposure limits and methods to minimize radiation exposure. World Neurosurg 82:13371343,2014

35. Sukovich W, Brink-Danan S, Hardenbrook M: Miniature robotic guidance for pedicle screw placement in posterior spinal fusion: early clinical experience with the SpineAssist. Int J Med Robot 2:114-122, 2006

36. Tsai TH, Wu DS, Su YF, Wu CH, Lin CL: A retrospective 
study to validate an intraoperative robotic classification system for assessing the accuracy of Kirschner wire (K-wire) placements with postoperative computed tomography classification system for assessing the accuracy of pedicle screw placements. Medicine (Baltimore) 95:e4834, 2016

37. van Dijk JD, van den Ende RP, Stramigioli S, Köchling M, Höss N: Clinical pedicle screw accuracy and deviation from planning in robot-guided spine surgery: robot-guided pedicle screw accuracy. Spine (Phila Pa 1976) 40:E986E991, 2015

38. Verma SK, Singh PK, Agrawal D, Sinha S, Gupta D, Satyarthee GD, et al: O-arm with navigation versus C-arm: a review of screw placement over 3 years at a major trauma center. Br J Neurosurg 30:658-661, 2016

39. Xia L, Wang X, Xu T, Zhang X, Zhu Z, Qin L, et al: Robotic versus open radical cystectomy: an updated systematic review and meta-analysis. PLoS One 10:e0121032, 2015

40. Yu E, Khan SN: Does less invasive spine surgery result in increased radiation exposure? A systematic review. Clin Orthop Relat Res 472:1738-1748, 2014
41. Zihni AM, Ohu I, Cavallo JA, Cho S, Awad MM: Ergonomic analysis of robot-assisted and traditional laparoscopic procedures. Surg Endosc 28:3379-3384, 2014

\section{Disclosures}

Dr. Park is a consultant for and receives royalties from Globus. He is also a consultant for Medtronic, Zimmer-Biomet, and NuVasive.

\section{Author Contributions}

Conception and design: Park. Acquisition of data: all authors. Analysis and interpretation of data: all authors. Drafting the article: Joseph, Smith. Critically revising the article: Park, Joseph. Reviewed submitted version of manuscript: all authors. Study supervision: Park.

\section{Correspondence}

Paul Park, Department of Neurosurgery, University of Michigan, 1500 East Medical Center Dr., Rm. 3553 TC, Ann Arbor, MI 48109-5338. email: ppark@med.umich.edu. 